\title{
Advanced Titanium Alloy Fatigue Modeling
}

Michael G. Glavicic1, Tom Broderick2, Vasisht Venkatesh3, Iuliana Cernatescu3, Daira Legzdina4, Fatmata Barrie5, Elizabeth Burns5, Ian Demptster6, Vikas Saraf7, Kayla Calvert8, Seshacharyulu Tamirisakandala9, Ravi Shankar10, Ayman Salem11, Somnath Ghosh12, Jon Almer13, William Mulsinski14, and Paul Shade14

1Rolls-Royce Corporation, Indianapolis, IN

2General Electric Aviation, Cincinnati, $\mathrm{OH}$

3Pratt \& Whitney, Hartford, CT

4Honeywell Aerospace, Phoenix, AZ

5The Boeing Company, St Louis, MO

6PCC - Wyman-Gordon, Houston, TX

7ATI Forged Products, Cudahy, WI

8Titanium Metals Corporation (TIMET), Henderson, NV

9Arconic, Niles, $\mathrm{OH}$

10Scientific Forming Technologies, Columbus, $\mathrm{OH}$

11Materials Resources LLC, Dayton, $\mathrm{OH}$

12Johns Hopkins University, Baltimore, MD

14Air Force Research Laboratory, Materials and Manufacturing Directorate,

AFRL, Wright-Patterson AFB, OH

\begin{abstract}
$\underline{\text { Abstract }}$
A summary of the final progress achieved in two Metals Affordability Initiative (MAI) programs (RR-12 and RR-13) that were funded by the US Air Force to develop the necessary integrated computational materials engineering (ICME) framework, knowledge, and supporting database to model and predict location-specific fatigue properties across the entire titanium supply chain is presented. Validation of this ICME framework which allows for the prediction of location specific low cycle fatigue (LCF) and high cycle fatigue (HCF) behavior on complex production components in electro-polished and shot peened surface conditions will be presented. In addition, validation of a new shot peening capability embedded with the commercial software package DEFORMTM will be presented.
\end{abstract}

\section{Introduction}

The results of the two MAI programs described herein were follow-on programs to three previously funded MAI (LAD-2, LAD-4 and RR-10) programs which were aimed at developing and demonstrating titanium microstructure and mechanical tensile property models on the most widely used titanium alloy, Ti-6Al-4V. Under these previous programs 1,2, models were developed to predict the crystallographic texture, key microstructural features (primary alpha size and volume fraction, beta grain size, grain boundary alpha thickness, and lamellar alpha thickness) and tensile properties in a unified package (Figure 1a).

At the conclusion of these successful programs, follow-on work was conducted to develop the necessary databases and models under the same framework (Figure 1b) that would enable the prediction of fatigue properties on electro-polished (RR-12) and shot peened specimens (RR-13). The changes made to DEFORMTM constituted both the inclusion of several new models within DEFORM ${ }^{\mathrm{TM}}$ that allowed shot peening process to be modeled as well as the addition of links to externally developed executable codes that are called by DEFORM ${ }^{\mathrm{TM}}$ to predict location specific fatigue properties based upon the microstructure and crystallographic texture developed in forged Ti-6Al-4V. In the sections to follow, a brief description of the models and plug-ins developed are described.

\section{Materials and Experiments}

In order to separate the effects of surface condition (shot peening) from the effects of microstructure and crystallographic texture, fatigue test databases were developed using identical test materials for the RR-12 and RR-13 programs. For the low cycle fatigue (LCF) portion of these databases, 6" and 10" billet material from two primary metal producers was forged into pancakes by two separate forging houses and solution heat treated in two commonly used conditions. For the high cycle fatigue (HCF) portion of these databases, specimens were tested from: 1) commercial blisk and blade components, 2) extruded6" billet and then side pressed, and 3) rolled plate materials. The conditions used to generate the test specimens to populate both the low cycle fatigue (LCF) and high cycle fatigue (HCF) databases were selected to best emulate the standard practices of the US aerospace industry. From the approximately thousand specimens generated using the procedures described above, two types of surface conditions were examined: 1) an electro-polished condition which would best emulate the intrinsic materials capability based upon the specimens microstructure and crystallographic texture and 2) electropolished plus shot peened specimens to examine the effects of imposing a near surface residual stress on the specimens fatigue properties. Specifically for the second set of specimens which were tested after shot peening, two shot peening conditions were examined (3A and 6A) for the LCF portion program, whereas for the HCF portion, three peening conditions 
(3A, 6A and 9N) were examined. All LCF tests for both the electro-polished or shot peened surface condition were conducted in strain control in a $\mathrm{R}=0$ test condition over a variety of strain levels with a total of 7 to 15 specimens tested for each microstructure, crystallographic texture or surface conditions. In addition LCF specimens were tested in both the hoop and axial directions to ascertain the effects crystallographic texture. For the HCF portion of the test program, the specimens were tested under load control in $\mathrm{R}=0,-1$ and 0.6 test conditions at loads that emulate the standard practice for $\mathrm{HCF}$ testing by the US aerospace supply chain. Once again HCF specimens were tested in both the electro-polished and shot peened conditions for a variety of applied loads and specimens orientation directions (hoop and axial).

a)
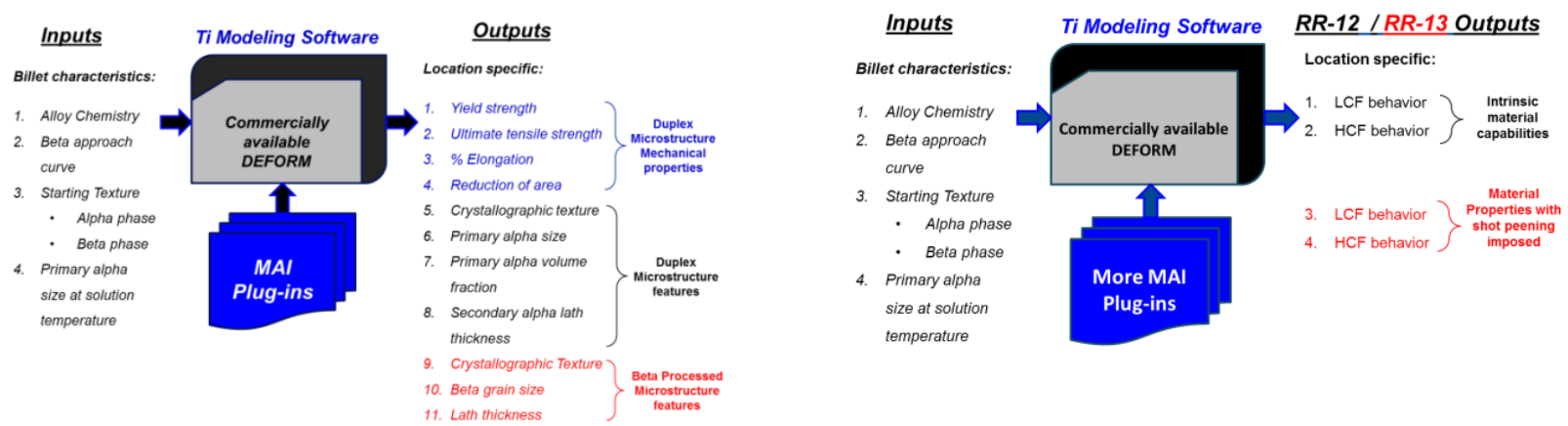

b)

Figure 1. High-level overview of a) the Advanced Titanium Alloy Microstructure and Mechanical Property Modeling program and b) the Advanced Titanium Alloy Fatigue Modeling programs (RR-12 and RR-13).

\section{Fatigue model database results}

Low cycle fatigue (LCF) testing of the electro-polished specimens revealed that when the strain controlled data was plotted on a semi-semi logarithmic plot very little scatter in the test data was present for a given microstructure or crystallographic texture present in the underlying set of test specimens (Figure 2). When identical specimens were tested in either the $3 \mathrm{~A}$ or $6 \mathrm{~A}$ shot peened condition, the slope and intercept of the fits of the data to a semi-logarithmic equation simply changed the slope and intercept of the data in a repeatable fashion for both the 3A and 6A shot peening conditions (Figure 2). Moreover the behavior of the $3 \mathrm{~A}$ and $6 \mathrm{~A}$ peened surfaces were found to behave effectively the same even though the near surface stress state imposed by the peening procedures on these two specimen sets were markedly different (Figure 3 ).

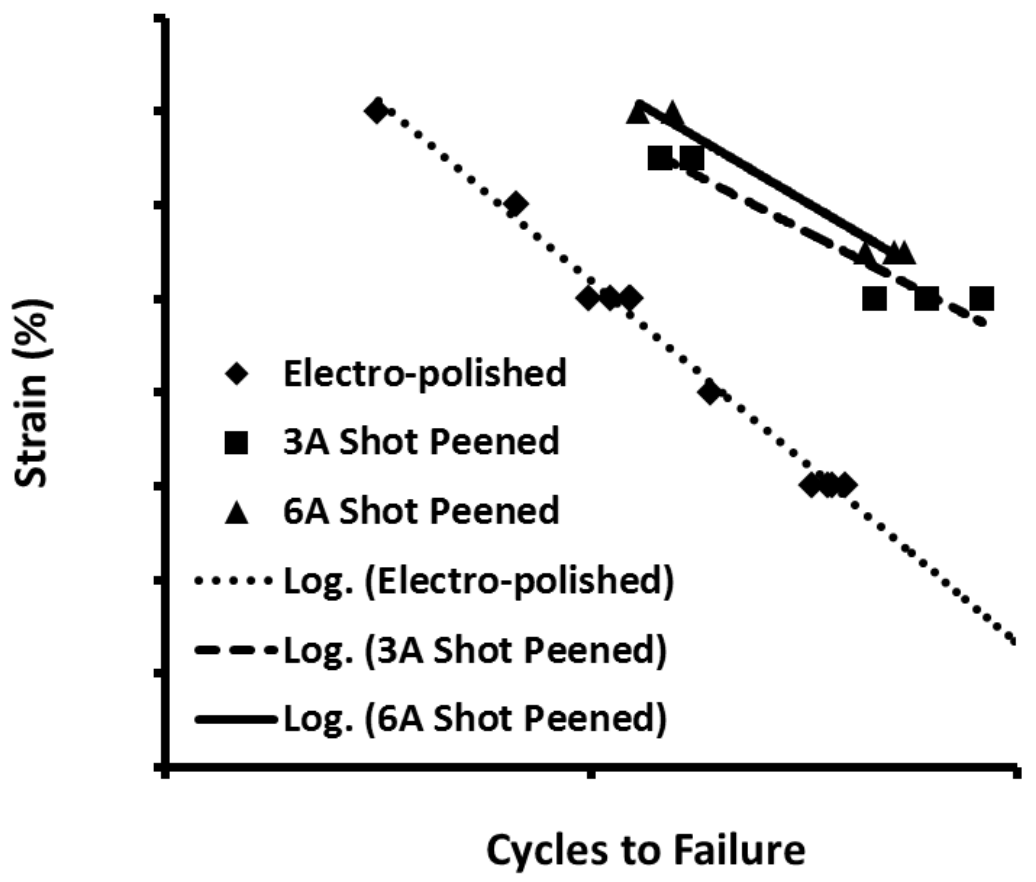

Figure 2. Typical low cycle fatigue (LCF) results obtained in the Advanced Titanium Alloy Fatigue Modeling programs for identical specimens that were electro-polished (RR-12) and shot peened (RR-13). 


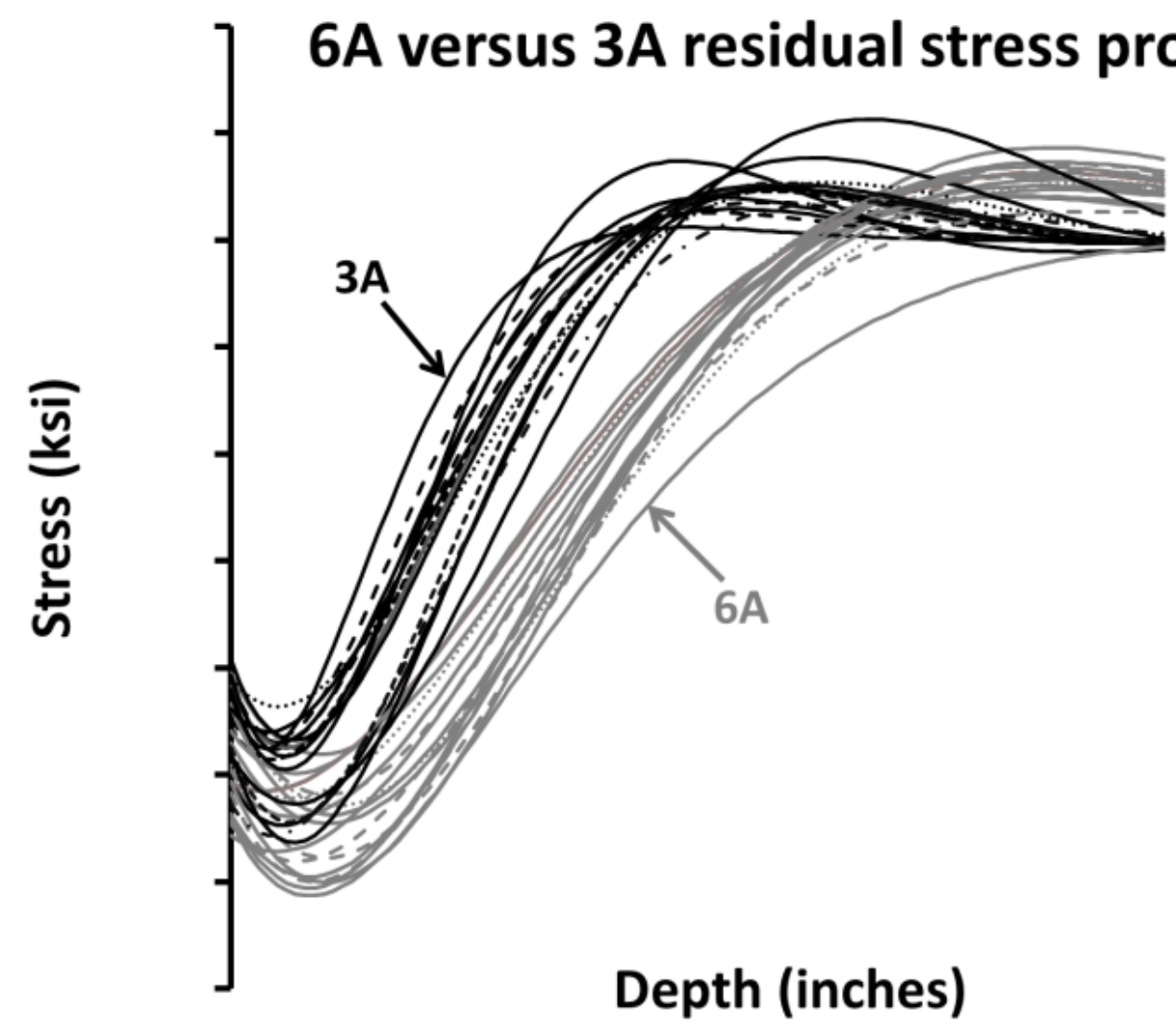

Figure 3. Fit of measured residual stress profiles for shot peening intensities of $3 \mathrm{~A}$ and $6 \mathrm{~A}$.

When the slopes and intercepts for all of the LCF data sets measured, from all of the microstructures and textures examined, were cross plotted against one another, all of the electro-polished data was found to lie on one line, whereas all of the test data for the shot peened specimens were found to lie on a second line displaced from the electro-polished data (Figure 4). This systematic behavior in the LCF data based upon the specimens underlying microstructure and crystallographic texture made it possible to develop models that would predict the low cycle fatigue (LCF) behavior of materials based upon their microstructure, crystallographic texture and surface condition (electro-polished versus shot peened). 


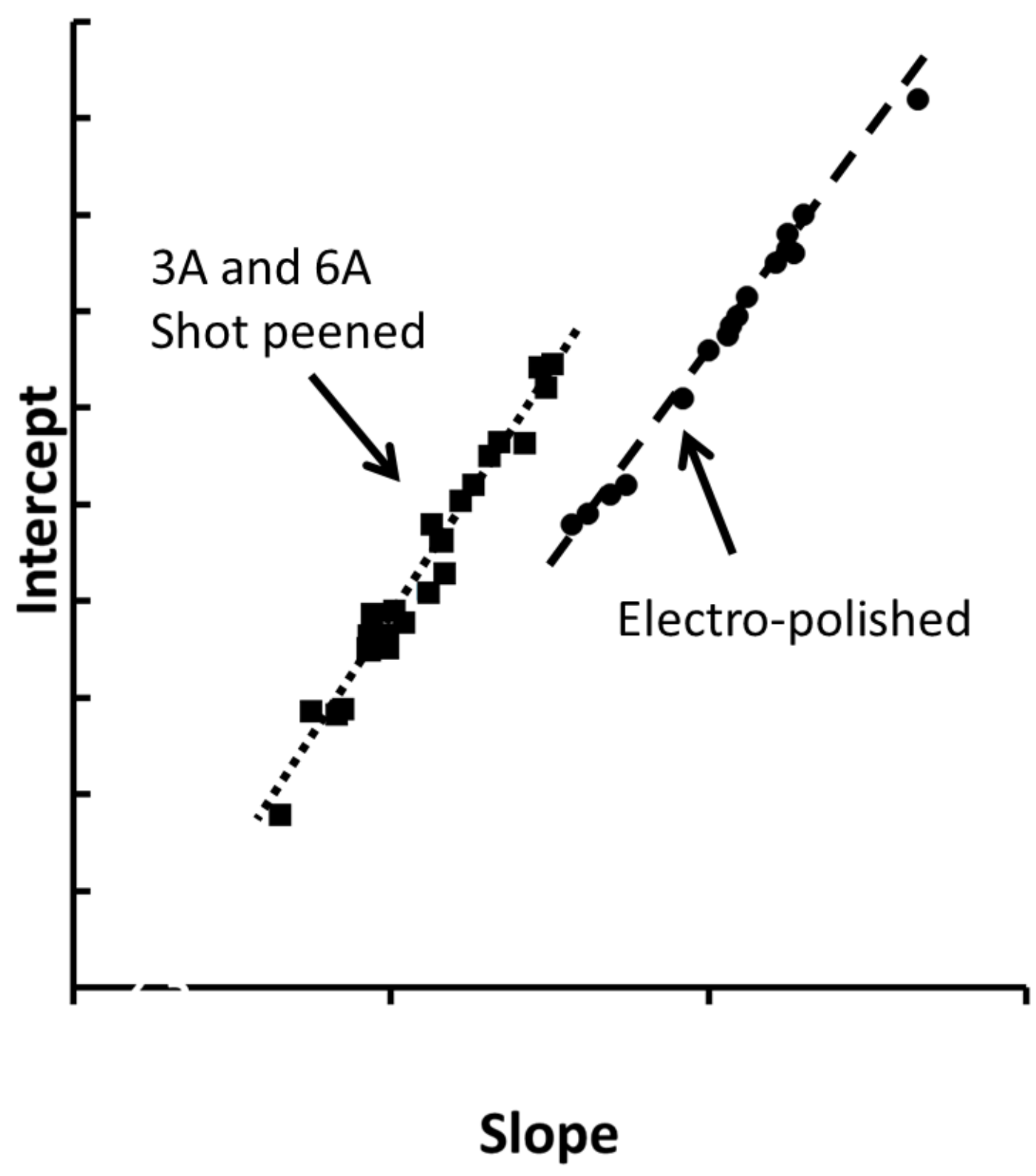

Figure 4. Cross plot of the fitted slopes and intercepts of low cycle fatigue (LCF) data collected electro-polished (RR12) and shot peened (RR-13) specimens.

In a similar fashion when the microstructure, crystallographic texture and surface condition were tightly controlled in load control high cycle fatigue (HCF) testing very little scatter in the test data was again observed. In this case the HCF test results for each set of electro-polished specimens with a specific microstructures, crystallographic texture and test direction was then fit to a power law $\left({ }^{\circ}=\mathrm{AN}^{\wedge} \mathrm{B}\right)$ in which "O " is the applied load, "N" is the number of cycles to failure, and " $\mathrm{A}$ " and "B" are fitting parameters for the dataset. When the power law fitted coefficients (A and B) for the HCF data sets from a variety of microstructures and crystallographic textures are cross plotted for test conditions of $\mathrm{R}=0,0.6$ and -1 , a clear linear trend is observed for all test conditions $(\mathrm{R}=0,-1$ and 0.6$)$ in which the specimens microstructure, crystallographic texture and specimen orientation were varied (Figure 5). This once again allowed a model to be developed to predict the HCF behavior based upon the specimens microstructure and crystallographic texture. Due to space limitations of this publication the validation results of the HCF model will be discussed in a future publication. 


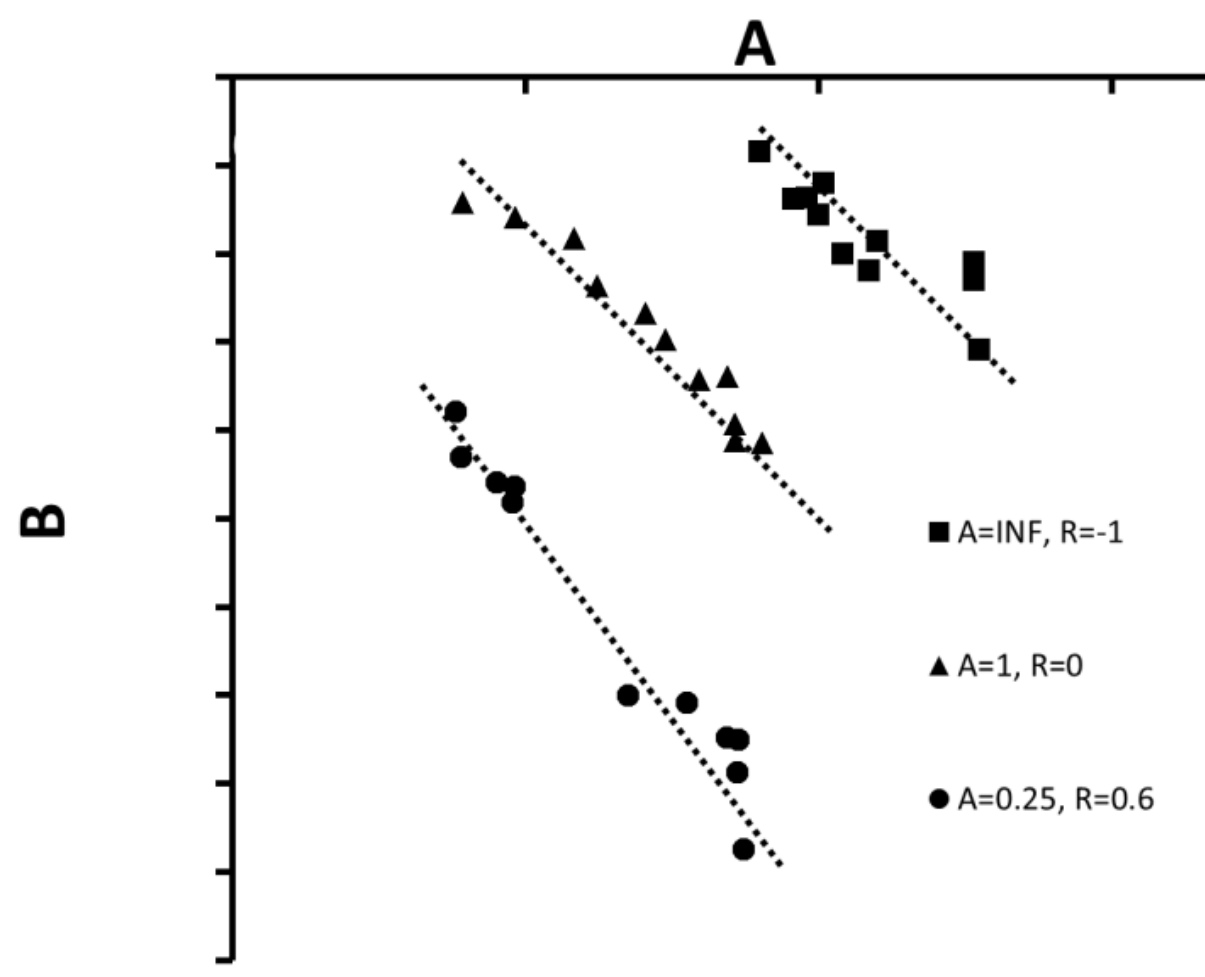

Figure 5. Cross plot of the fitted power law coefficients A and B of high cycle fatigue (HCF) data collected on electro-polished (RR-12) specimens.

\section{LCF Model validation results}

Validation of the LCF models developed for electro-polished and shot peened surfaces was performed using the same microstructures and crystallographic textures predicted on a Rolls-Royce production component which was used to validate the RR-10 models described at the previous World Titanium Conference held in 2015. In this previous work, the crystallographic textures and microstructures were used to predict the location specific tensile properties. In the current efforts, the same workflow described in Figure 1a was used to predict the necessary inputs for the LCF model (Figure 1b) at these same three locations previously examined1. This modeling work to determine the location specific microstructure and crystallographic texture was performed by PCC Wyman-Gordon which is the forging house which supplies Rolls-Royce with its condition of supply material for the production component examined here. As a result the modeling parameters used as input to the models represent the proprietary forging and heat treatment conditions developed by PCC Wyman-Gordon and constitute the boundary conditions and practices used in the fixed process approved by Rolls-Royce as part of its quality assurance practices. Hence the model validation results constitute the best possible result with the models developed because the model inputs used were the proprietary values to which Rolls-Royce has no knowledge of. This validation effort also represented how members of Metals affordability teams interact with one another for the purpose of deploying integrated computational materials engineering (ICME) frameworks across the entire US aerospace supply chain. Due to space restrictions for this conference publication, the results of only one location will be reported here, however the results discussed are representative of the results obtained at the other two locations examined in the validation efforts.

At location R1 of the forging, a total of nine strain controlled LCF tests were conducted on specimens with an electropolished surface finish (Figure 6). The range of strains selected for testing encompassed the typical LCF range used for Ti$6 \mathrm{Al}-4 \mathrm{~V}$ wrought specimens. The test results again displayed very little scatter due to the microstructure and crystallographic texture being tightly controlled. From this test data, error limits for three standard errors were calculated based upon the fit of the LCF test data to a semi-logarithmic function. The modeled low cycle fatigue behavior was then found to overlay the measured experimental data and to be inside of the three standard error limits that were calculated form the measured data. This result clearly demonstrated that the workflow deployed would allow for location specific LCF behavior to be predicted based upon the local crystallographic texture and underlying microstructure with the use of the tools developed under the RR-12 MAI program and cooperation across the tiers of the US aerospace supply chain. 


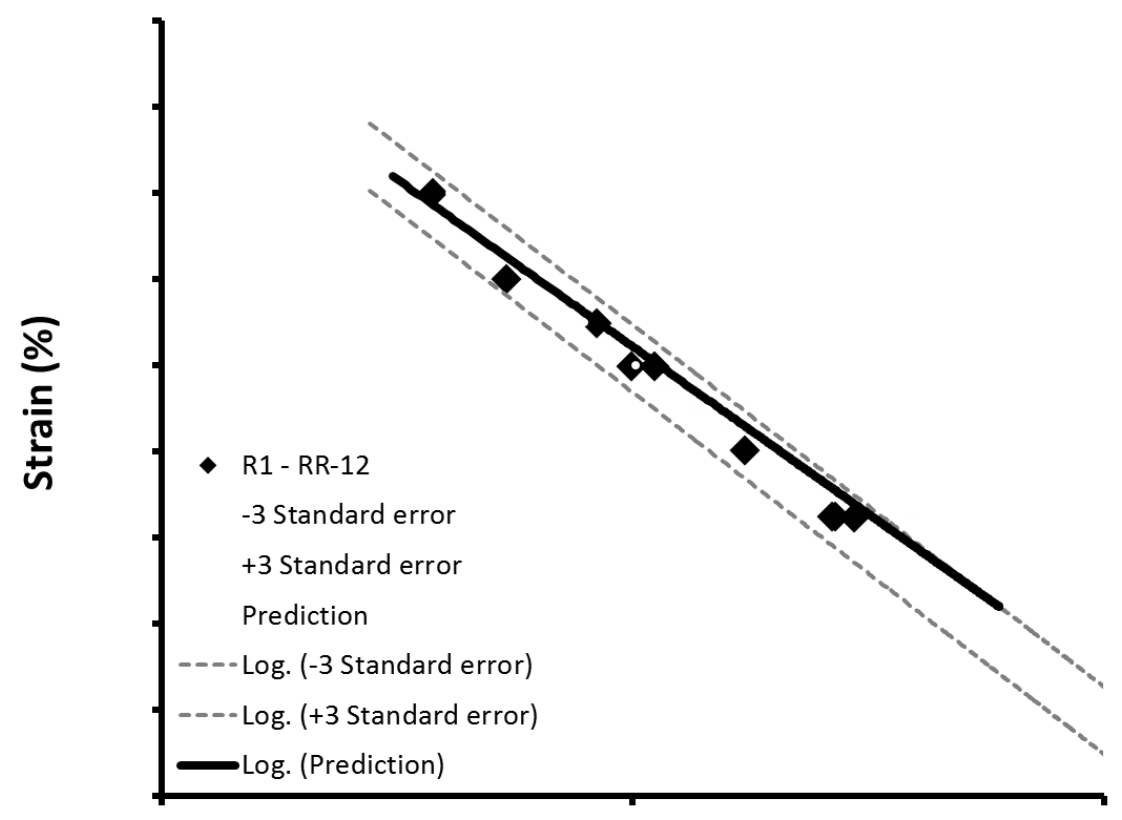

\section{Cycles to failure}

Figure 6. LCF model validation results on Rolls-Royce production forging on specimens whose surfaces were electro-polished (RR-12).

In order to test the LCF model developed for shot peened surfaces, a duplicate set of test specimens were tested in which their final surface conditions were shot peened after being electropolished. Once again three locations were examined in this work, but due to space limitiation of this publication the results of a single location will be disucssed, with this location being the same as that was studied in the electropolished surface condition. The behavior of these shot peened specimens in relation to the electropolished specimens (which were identical in microstructure and crytallogrpahic texture) demonstrated that the application of a shot peened surface to the LCF specimens, simply modified the slope of the LCF curve in relation to the behavior of the electroplolished specimens (Figure 7). Moreover for high applied strains in the LCF testing conducted the two curves began to intersect indicating that at high strains the benefts of shot peeinging disappear and the intrinsic capabality of the underlying microstructure and crystallographic texture is the mechanism that drives the fatigue behavior of the specimens. 


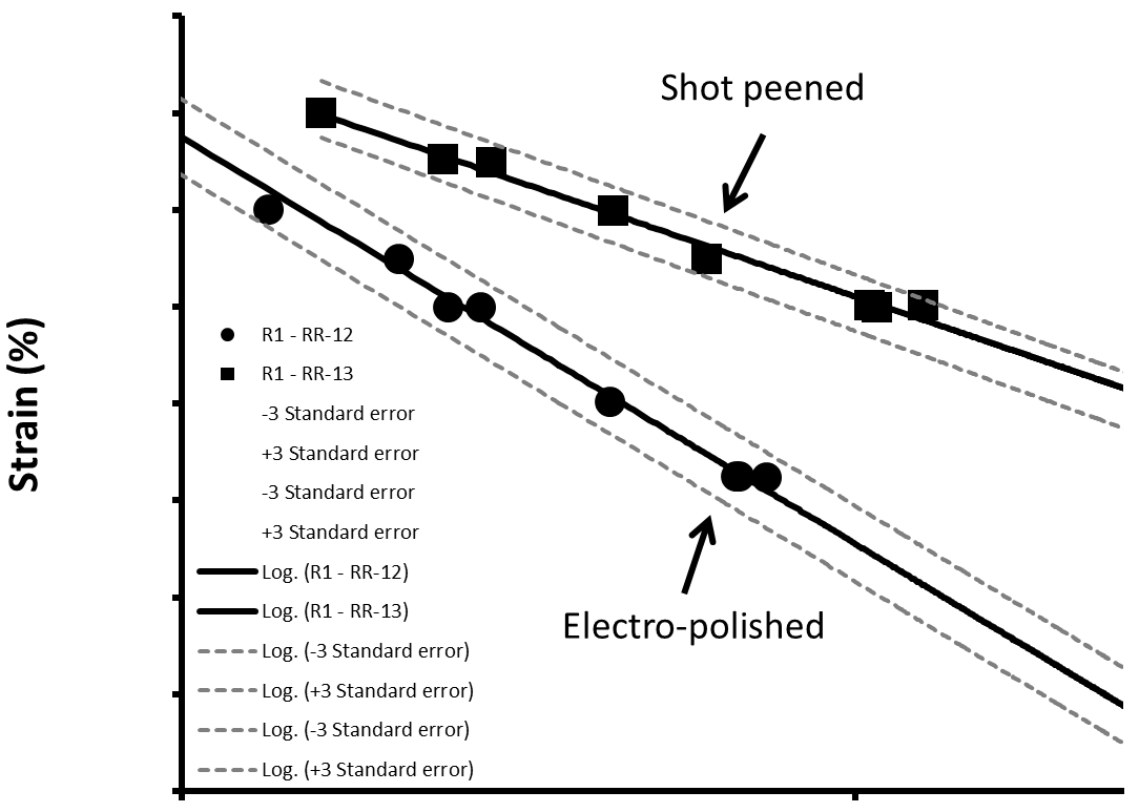

\section{Cycles to failure}

Figure 7. Low cycle fatigue (LCF) results obtained in the Advanced Titanium Alloy Fatigue Modeling programs for Rolls-Royce production forging for location R1 that were electro-polished (RR-12) and shot peened (RR-13).

In order to validate the LCF modeling capabilities with a shot peened surface, a total of eight strain controlled LCF test were conducted on specimens from location R1 in the Rolls-Royce production forging (Figure 8). Again the strains selected for testing encompassed the typical LCF range used for Ti-6Al-4V wrought specimens. The test results again displayed very little scatter due to the microstructure and crystallographic texture being tightly controlled. From this test data, error limits for three standard errors were again calculated based upon the fit of the LCF test data to a semi-logarithmic function. In addition because the model data did not in this case overlay the measured data, error limits with a $95 \%$ confidence index were also calculated based upon the fit of the LCF test data to a semi-logarithmic fit of the experimental data. The model results were then plotted and found to lay for the most part within three standard errors of the test data with a $95 \%$ confidence index (Figure 8). Once again this result clearly demonstrated that the workflow deployed would allow for location specific LCF behavior to be accurately predicted with the use of the tools developed under these MAI programs through the cooperation of several tiers of the US aerospace supply chain. 


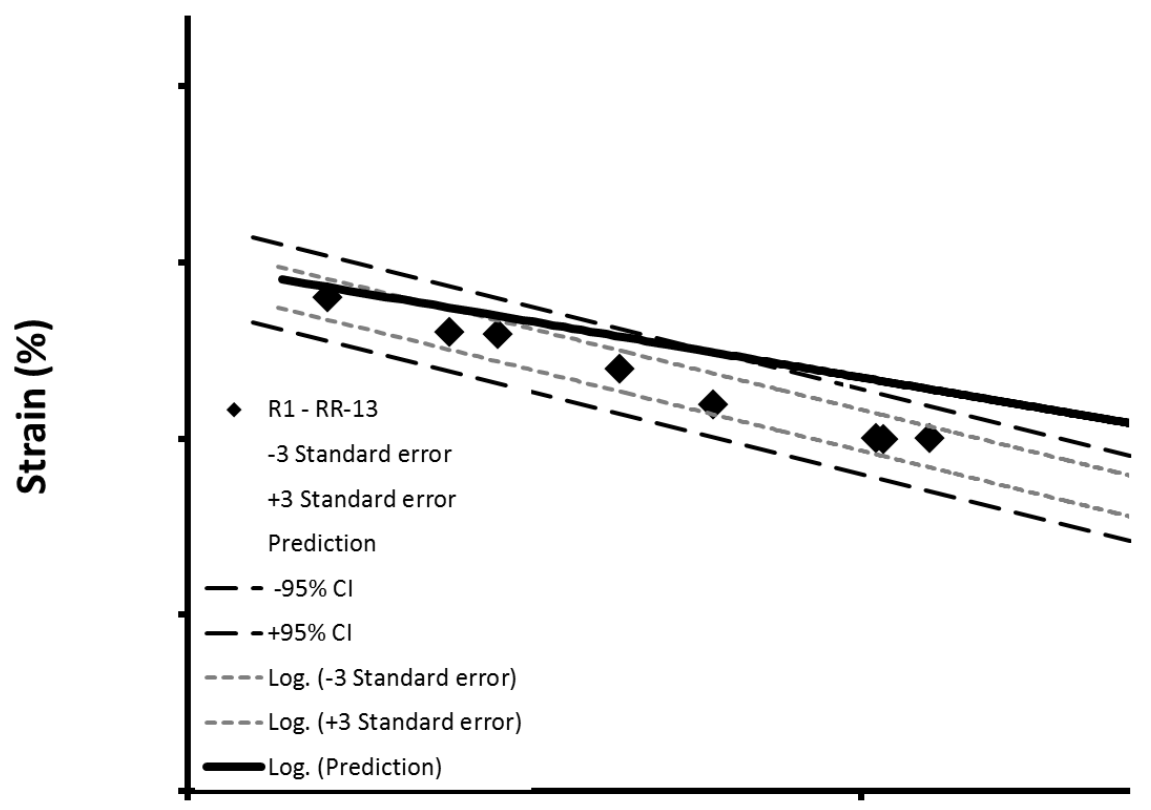

\section{Cycles to failure}

Figure 8. LCF model validation results on Rolls-Royce production forging on specimens whose surfaces were shot peened.

In a separate effort the modeling capabilities within the software package entitled DEFORM ${ }^{\mathrm{TM}}$ were enhanced in a manner to allow users of the software to predict the shot peening coverage and imposed residual stress profile as a result of a set of prescribed shot peening instructions. The validation of the peening models developed involved the 3D modeling of a peening procedure applied to a Pratt \& Whitney production blisk using several alternative peening strategies which varied the manner in which the peening nozzle was positioned and moved over the component. The validation of this newly developed peening capability was based upon the peening models ability to predict the residual stress profile imposed to within 3 standard errors of typical scatter in residual stress measurements performed by x-ray diffraction. The result of the validation work conducted at on key location investigated for one peening strategy investigated demonstrated that the models developed were able to predict the imposed residual stress profile to within 3 standard errors (Figure 9).

a)

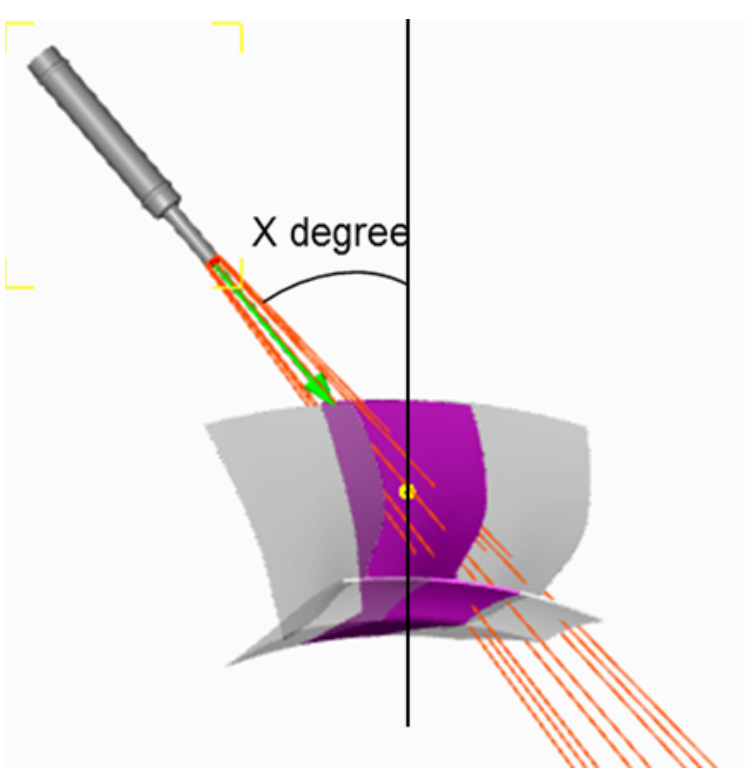

b)

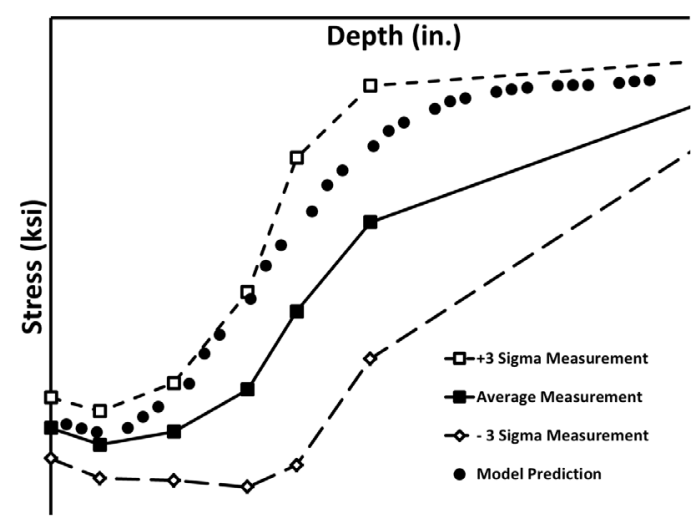


Figure 9. Validation of DEFORMTM shot peening model: a) schematic of a peeing procedure investigated and b) measured and simulated residual stress profile with respect to 3 standard errors of a residual stress profile measured using $x$-ray diffraction.

\section{Conclusions}

Achievements in the Advanced Titanium Fatigue Modeling programs (RR-12 and RR-13) funded under the Metals Affordability Initiative (MAI) were presented. The programs were successful in the development of a validated set of computational models that predict location-specific low cycle fatigue behavior based upon local microstructure and crystallographic texture in wrought titanium alloy components. In addition, the programs were also successful in developing new shot peening modeling capabilities with the commercial finite element software package called DEFORM ${ }^{\mathrm{TM}}$.

Finally, the results presented herein describe the culmination of the combined efforts of a large cross section of the US aerospace supply chain, academics, commercial vendors and the United States Air Force under the Metals Affordability Initiative (MAI) projects that have spanned two decades of effort. This modeling work described herein is the result of the knowledge and hard work performed under the funding of five separately funded titanium modeling programs (LAD-2, LAD-4, RR-10, RR12 and RR-13) and represents both a long term and large financial commitment by all parties involved. The results of the combined efforts of all of the parties of each of these MAI programs has led to the success outlined in the current publication and those presented at the previous World Titanium Conferecne1. Furthermore the work described in these efforts demonstrates that in order for integrated computational materials engineering (ICME) to become a reality in its use within the US aerospace industry a long term presence and continued funding for multiple programs is required to perform all for the detailed technical work required to model location specific properties within wrought Ti-6Al-4V. It further demonstrates that long term commitments by government agencies such as the United States Air Force and cooperative efforts from all members of large consortiums such as the Metals Affordability Initiative (MAI) are enablers for the development of integrated computational materials engineering (ICME).

\section{Acknowledgements}

The authors would like to acknowledge the support of the U.S. Air Force through the Metals Affordability Initiative (contracts FA8650-10-2-5219, FA8650-15-2-5203 and FA8650-15-2-5223).

\section{$\underline{\text { References }}$}

1. M.G. Glavicic, et al., "Progress in the Advanced Titanium Microstructure and Modeling Program", Proc. 13th World Conference on Titanium, ed. V. Venkatesh, et al. (Warrendale, PA, 2015) pp 1867-1873.

2. M.G. Glavicic and V. Venkatesh, "Integrated Computational Materials Engineering of Titanium: Current Capabilities Being Developed Under the Metals Affordability Initiative”, JOM, Volume 66, Issue 7, pp.1310-1320 